\title{
Latest results of the measurement of the Higgs boson decaying to bottom quark pair
}

\author{
Bortignon P.* on behalf of the CMS Collaboration \\ University of Florida \\ E-mail: pierluigi.bortignonegmail.com
}

The latest results of the measurement of the Higgs boson decaying to bottom quark pair are presented in this proceedings. The analyses are performed using data collected with the CMS experiment in 2016 and 2017 at the LHC from pp collisions at centre-of-mass energies of $13 \mathrm{TeV}$.

ICHEP 2018, International Conference on High Energy Physics

4-11 July 2018

Seoul, South Korea

${ }^{*}$ Speaker. 


\section{Introduction}

These proceedings are dedicated to review the most recent result on the Higgs boson decaying into a bottom-quark pair, using data produced by proton-proton collision at the Large Hadron Collider (LHC), and collected by the CMS detector. The CMS detector is described in details in [1].

The Higgs boson was discovered at the LHC together by the CMS and ATLAS Collaboration $[2,3]$ in July 2012. Evidence of the Higgs boson decay to WW, ZZ, $\gamma \gamma$, and $\tau \tau$ is established established[2, 5, 4]. The Higgs boson larger decay rate is to pairs of b-quarks, with a branching ratio of 58\%. However the dataset collected so far was not large enough to observe this decay mode.

The $\mathrm{H} \rightarrow \mathrm{b} \overline{\mathrm{b}}$ is as important to understand the standard model and the Higgs sector as it is for discovering new physics. CMS analyses cover all the main Higgs boson production mechanisms at the LHC. The analyses presented in this proceedings are the associated production with a vector boson, the golden channel at LHC for $\mathrm{H} \rightarrow \mathrm{b} \overline{\mathrm{b}}$ searches and measurements, and the associated production with a single top, the most recent addition to the $\mathrm{H} \rightarrow \mathrm{b} \overline{\mathrm{b}} \mathrm{CMS}$ analyses. The latter has direct access to the top quarks coupling of the Higgs boson and to its sign, making this analysis unique despite its low sensitivity to the SM Higgs boson signal. In case of top-quark inverted coupling the cross section of the its production mechanism is strongly enhanced.

\section{2. tH, Hbb}

The production of single top + Higgs $(\mathrm{tH})$ allows to probe the direct coupling of the Higgs boson to the top quark via two main topologies: tHq and tHW.

The analysis is targeting both production mechanisms, tHq and tHW. It is also sensitive to the Higgs production in association with top quark pairs $(\mathrm{ttH})$. The analysis is divided in two signal categories according to the number of b-jets in the event. It includes also a third category with two leptons, to have a better control of the dominant $t \bar{t}+$ heavy flavor background.

Multivariate analysis techniques are used in several stages of the analysis. A boosted decision tree (BDT) is trained to resolve the combinatorics of the jet assignment. This discriminator is then used as input to another BDT trained to separate the signal from the t⿱亠t background. Another BDT is used to separate the $t \bar{t}+$ light flavor from the $t \bar{t}+$ heavy flavor events in the dileptonic background control region.

The results are presented in Fig. 1(Left) as 95\% confidence level upper limit on the cross section times branching ratio, as a function of the ratio between the top and vector boson coupling modifier, $\kappa_{\mathrm{t}}$ and $\kappa_{\mathrm{V}}$. The wiggle in the limit between $\kappa_{\mathrm{t}} / \kappa_{\mathrm{V}}=-1$ and 1 shows the analysis sensitivity to the three production mechanisms: for coupling ratios close to $\kappa_{\mathrm{t}} / \kappa_{\mathrm{V}}=-1$, the diagram interference for both tHq and tHW is constructive and the tH cross sections become comparable to $\mathrm{ttH}$. When the ratio reaches $\kappa_{\mathrm{t}} / \kappa_{\mathrm{V}}=0$, the $\mathrm{ttH}$ cross section vanishes and only the $\mathrm{tH}$ processes contribute. At a value of $\kappa_{\mathrm{t}} / \kappa_{\mathrm{V}}=1$, the standard model prediction, the tHq and tHw cross sections are small and $\mathrm{ttH}$ is the dominant contribution. 


\section{VH, Hbb}

The associated production with a vector boson is the most sensitive analysis at the LHC for $\mathrm{H} \rightarrow \mathrm{b} \overline{\mathrm{b}}$.

The CMS VHbb analysis described in [6] is divided in three main channels, depending on the number of isolated leptons present in the event.

The dominant backgrounds for the high sensitivity categories are the associated production of a vector boson with heavy flavour jets, and top-quark pair production. Control regions in the signal sidebands are used to study and control the main backgrounds.

Event are categorized as a function of vector boson transverse momentum, which in signal events is harder than in events produced from the main backgrounds. This categorization isolates higher signal-over-background categories, increasing the analysis sensitivity.

The invariant mass resolution is an important variable in the analysis for distinguishing signal and background. Improving the invariant mass resolution is directly beneficial to the sensitivity of the analysis. Jet energy scale and resolution are measured centrally by CMS in an unbiased way in bins of $\eta$ and $\mathrm{p}_{\mathrm{T}}$ [7]. Dedicated corrections for b-jets in the phase space of interested are derived using a regression trained using jets from $t \bar{t} \mathrm{MC}$ simulation. After the application of the corrections the invariant mass resolution shows an overall improvement of about $15 \%$, which increases when looking at events with muons inside b-jets.

A machine learning algorithm is used to separate the signal from the large background from $\mathrm{V}+\mathrm{jets}$ and $\mathrm{t} \overline{\mathrm{t}}$. Variables discriminating signal from background, including the Higgs candidate mass, are passed to a BDT for training. The BDT is trained with events passing loose preselection useful to reduce the background while keeping the signal efficiency high. Background contamination in the signal region is estimated using MC predictions corrected on data in signal sidebands control regions. The BDT discriminator shape is used for signal extraction using a template fit.

An important validation of this analysis strategy and tools is the extraction of the $\mathrm{VZ} \rightarrow$ $\mathrm{b} \bar{b}$ resonant signal, that shares several characteristic with the signal. The observed significance for these events is above 5 sigma, and the expected yield compatible with the SM expectation.

The observed significance of the SM Higgs boson is 3.8 sigma, and the measured signal strength modifier is compatible with the standard model within an uncertainty of 30\%. Fig. 1(Right) shows the excess of data events compared to the background only expectation and compatible with a VH(bb) signal.

\section{Summary}

Tab. 1 shows the latest results from all the $\mathrm{H} \rightarrow \mathrm{b} \overline{\mathrm{b}}$ analyses using LHC data collected by the CMS detector. The ttH production mode is the single most sensitive production mechanism. The $\mathrm{VH}$ combination, including the $\mathrm{W}$ and $\mathrm{Z}$ production mechanisms, is the most sensitive channel. The combined signal strength is compatible with SM expectation.

Measuring $\mathrm{H} \rightarrow \mathrm{b} \bar{b}$ is important for understanding the Yukawa interaction, and for searches for new physics. The larger dataset and improved analysis tools are increasing the analyses sensitivity. The $\mathrm{VH}$ production mechanism is the most promising channel for the first observation of the $\mathrm{H} \rightarrow$ $\mathrm{b} \overline{\mathrm{b}}$ decay. 

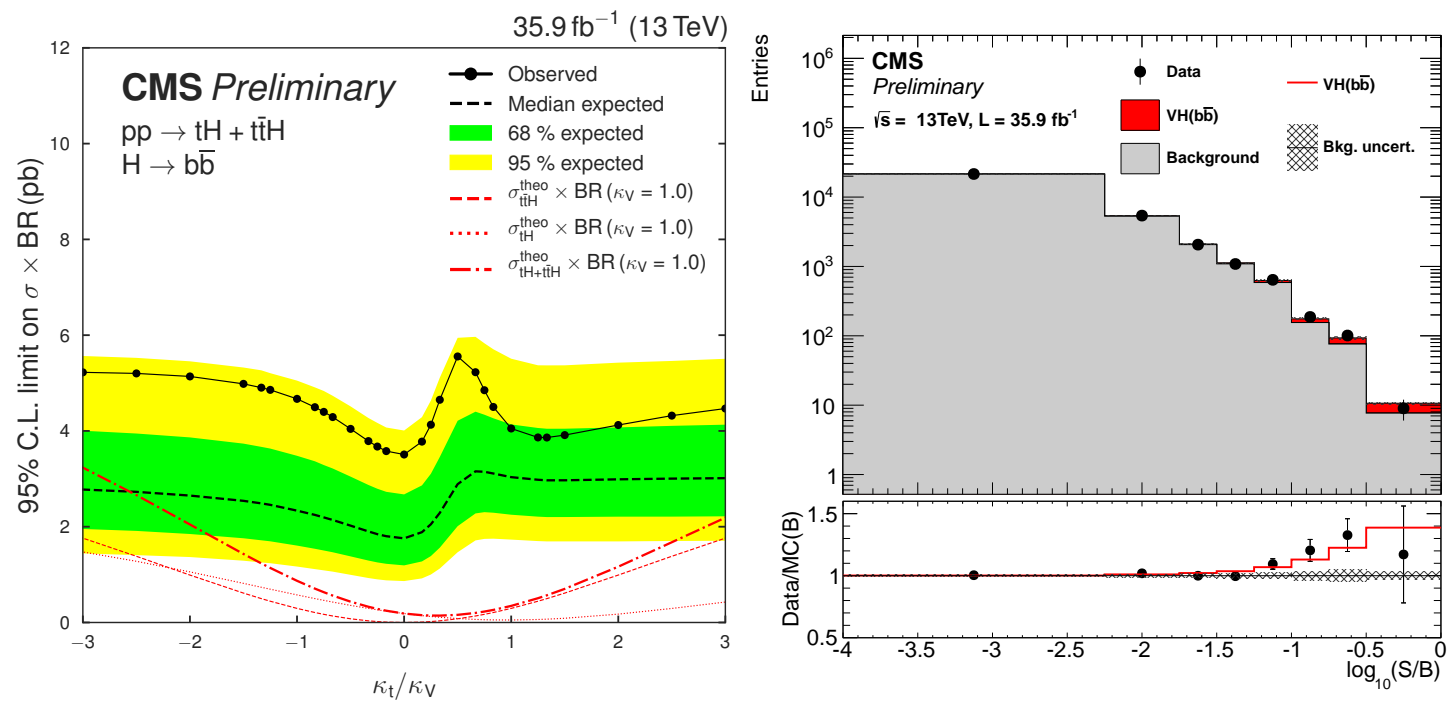

Figure 1: Left: Upper limits on $\mathrm{tH}+\mathrm{ttH}$ scenarios with different $\kappa_{\mathrm{t}} / \kappa_{\mathrm{V}}$ ratios. The red lines show the theory predictions and the inner (green) band and the outer (yellow) band indicate the regions containing 68 and $95 \%$, respectively, of the distribution of limits expected under the background-only hypothesis. None of the inspected points can be excluded. Right: Signal over background ratio of all categories for data and MC predictions showing an excess of data events compared to the background-only expectation compatible with a VZ(bb) (left) and VH(bb) (right) signal.

\begin{tabular}{l|l|l|l|l|l}
$\begin{array}{l}\text { Production } \\
\text { mechanism }\end{array}$ & dataset [TeV] & lumi $\left[\mathrm{fb}^{-1}\right]$ & 95\%CL limit & significance & signal strength \\
\hline \hline $\mathrm{ggH}_{+\mathrm{VBF}+\mathrm{VH}+\mathrm{ttH}}$ & 13 & 35.9 & $5.8(3.3)$ & $1.6(0.5)$ & $2.3_{-1.6}^{+1.8}$ \\
\hline $\mathrm{VH}$ & $7+8+13$ & $5.0+19.7+35.9$ & & $3.8(3.8)$ & $1.06_{-0.29}^{+0.31}$ \\
\hline $\mathrm{VBF}$ & $8+13$ & $19.8+2.32$ & $5.0(2.2)$ & $1.2(1.0)$ & $1.3_{-1.1}^{+1.2}$ \\
\hline $\mathrm{ttH}$ & $7+8+13$ & $5.0+19.7+35.9$ & & & $0.82_{-0.42}^{+0.44}\left(_{-0.42}^{+0.44}\right)$ \\
\hline $\mathrm{tH}[\mathrm{ITH}]$ & 8 & 19.7 & $7.6[5.4]$ & & \\
$\mathrm{tH}[\mathrm{ITH}]$ & 13 & 35.9 & $5.83[2.94]$ & &
\end{tabular}

Table 1: Summary table of all CMS Hbb searches latest results at the time of the conference. Values in brackets are expected values from SM theory with the exception of the tH analyses where the values in brackets correspond to the inverted top coupling (ITH) observed values.

\section{References}

[1] CMS Collaboration, JINST 3 (2008) S08004.

[2] CMS Collaboration, PLB 716 (2012) 30

[3] ATLAS Collaboration, Phys. Lett. B 716 (2012) 1

[4] CMS Collaboration, Phys. Lett. B 779 (2018) 283

[5] ATLAS and CMS Collaborations, JHEP 08 (2016) 045

[6] CMS Collaboration, Phys. Lett. B 780 (2018) 501

[7] CMS Collaboration, JINST 12 (2017) no.10, P10003 